Research Paper

\title{
Isolation and Characterization of Muscle Fatigue Sub- stance with Anti-Tumor Activities
}

\author{
Ruben M. Munoz, Haiyong Han, Tony Tegeler, Konstantinos Petritis, Daniel D. Von Hoff, Stanley A. \\ Hoffman $^{\bowtie}$ \\ Division of Clinical Translational Research, Translational Genomics Research Institute, Scottsdale, Arizona, USA
}

$\triangle$ Corresponding author: Stanley Hoffman, M.D. 445 N. Fifth St. Phoenix, AZ 85004. Email: stan@stanleyhoffman.com. Phone: 480-991-2269

(c) Ivyspring International Publisher. This is an open-access article distributed under the terms of the Creative Commons License (http://creativecommons.org/ licenses/by-nc-nd/3.0/). Reproduction is permitted for personal, noncommercial use, provided that the article is in whole, unmodified, and properly cited.

Received: 2012.10.19; Accepted: 2013.04.25; Published: 2013.05.09

\begin{abstract}
Research during the 1950's indicated that exercise played a role in the reduction of tumor growth. In the 1960's our studies confirmed that tumor-bearing rats, exercised to fatigue, demonstrated tumor inhibition. Our further studies isolated an extract (Fatigue Substance, or F-Substance) from rectus femoris muscles of rats which had been electrically stimulated to fatigue. This extract significantly inhibited growth of transplanted rat tumors. Research continued until 1978 when it became apparent the methodology at that time was not able to further identify the substance's active components. Using current technology, we now report on the further isolation and characterization of F-Substance. In cell proliferation assays, extracts from electrically stimulated rat rectus femoris muscles had more significant inhibitory effect on the breast cancer cell line MCF-7 than those isolated from unstimulated muscles. To identify the molecule(s) responsible for the antitumor activity, a rat cytokine antibody array was used to profile the cytokines in the substances. Among the 29 different cytokines contained on the array, 3 showed greater than 3 -fold difference between the substances isolated from the stimulated and unstimulated muscles. LIX (also known as CXCL5) is 6-fold higher in the substances isolated from stimulated muscles than those from the unstimulated muscles. TIMP-I is 4.6 fold higher and sICAM is 3.6 fold higher in the substances from the stimulated muscles. Our results indicated that cytokines released from contracting muscles might be responsible for the antitumor effect of F-Substance.
\end{abstract}

Key words: exercise, muscle fatigue substance, F-Substance, antitumor

\section{Introduction}

Experiments concerning the effect of exercise on cancer were reported during the 1950's. Heilbrunn and Rashkis obtained an increase in survival time in exercised tumor-bearing rats $(1,2)$. In 1959 we initiated a series of experiments to determine the influence of exercise on tumor growth. Rats were exercised by swimming and/or running, with results of all studies showing tumor reduction, with tumor weights in control rats exceeding that of the exercised rats in every instance $(3,4)$. During the 1960's and 70's our research continued with a series of experiments using an extract from rat rectus femoris muscles that been electrically stimulated to fatigue $(4,5,6)$. We injected this Fatigue Substance (F-Substance) into tumor-bearing rats and mice, causing a reduction in the size of all transplanted tumors, including several complete regressions, with no significant decrease in the carcass weight of the injected rats. Tumors used in the various studies were: Walker 246 and Murphy Lymphosarcoma in rats; Erlich and S37 mouse tumors in mice. Between 1969 and 1978 our studies on F-Substance were expanded to include determination of a bio-assay method, separation by Gel Filtration, biochemical separation, and determination of tumor- 
istasis in relation to voltage regulation in the exercised muscle $(7,8,9)$. We found that the extract inhibited tumor growth without involving surrounding tissue and could be injected into the tumor site or in the opposite side of the rat with no change in results. We theorized that the substance was a polypeptide with a molecular weight of $40 \mathrm{KDa}$ to $60 \mathrm{KDa}$. It was standardized by volume, rather than weight.

Research was discontinued in 1978 when it became apparent that methodology at that time was not sophisticated enough to identify the extract's active components. After a hiatus of 30 years, in this study we sought to isolate and characterize the components and activity of F-Substance using current technology. Confirming our original theory, we have shown that it contains peptides. Apoptotic signaling has been demonstrated in analyzing the peptides. Our analysis showed that the substance has significant antitumor activity and identified 3 cytokines that may be responsible for this anti-tumor activity.

\section{Material and Methods}

\section{Extraction of F-Substance}

To prepare the fatigue substance, we assembled a system that allows us to electrically stimulates rat rectus femoris muscles, monitor their contractions, and collect the substances that were released. The system is a modified version of the one used in our original studies (4). It consists of a stimulator (Model SD9, Grass Instruments), a force transducer (Biopac Systems Inc.), a data acquisition unit (Model MP35, Biopac Systems Inc.), and a water bath.

The right and left rectus femoris muscles were removed from 8-9 week old Wistar rats and rinsed with saline solution to remove any trace of blood on the surface. The muscles were clamped between two electrode clips that carried current from the stimulator. They were then placed in a conical tube containing $30 \mathrm{ml}$ of saline solution, which was placed in a $37^{\circ} \mathrm{C}$ water bath. After applying $30 \mathrm{~g}$ of force to the muscles by the force condenser, 9 volts of electricity were applied to one of the muscles for $180 \mathrm{sec}$ and the other muscle from the same rat was left in the saline unstimulated for the same period of time as a control. The contraction pattern of the stimulated muscle was recorded by the transducer. The saline solutions were then spun at 2,000 rpm for 5 minutes to remove blood cells. The samples were then dialyzed in $3.5 \mathrm{~K}$ MWCO dialysis cassettes (Pierce Biotechnology) against in 5 liters of $\mathrm{dH}_{2} \mathrm{O}$ changed four times. Samples were finally lyophilized at $-20^{\circ} \mathrm{C}$ until dry. Before proteomic analysis, the samples were reconstituted in $50 \mathrm{mM}$ ammonium bicarbonate. Initial proteomic experi- ments revealed high concentrations of blood related proteins despite of the washing of the muscle before it's stimulation. In order to decrease the concentration of blood proteins, the sample was further processed by using a Seppro mouse immunodepletion cartridge following by reduction, cysteine alkylation, and tryptic digestion. The digest was cleaned up by using Waters Sep-Pak solid phase extraction cartridges, lyophilized and stored at $-20^{\circ} \mathrm{C}$ until LC-MS/MS analysis.

\section{Evaluation of F-Substance using cancer cell line models}

To evaluate the anti-tumor activity of the substance released by the stimulated muscles, we measured its effect on the growth of two cancer cell lines: Mia PaCa-2 (a pancreatic cancer cell line) and MCF-7 (a breast cancer cell line). The cancer cells were seeded in 96-well cell culture plates at 2,000 cells/well and treated with different amounts of substances extracted from stimulated or unstimulated rat muscles for 72 hours. At the end of treatment period, cell viability was measured using the sulforhodamine B (SRB) assay (10). Each treatment was done in triplicates. P values were calculated using paired $t$ test.

\section{Evaluation of F-Substance by HPLC and Mass Spectrometry}

A Waters nanoAcquity UPLC system running in 1D mode combined with a Thermo LTQ Orbitrap Velos ETD operating in positive ion mode was used to analyze the sample. Peptides were analyzed by injecting $1 \mu \mathrm{g}$ of the digest onto a trap column (Symmetry C18 $5 \mu \mathrm{m} 180 \mu \mathrm{m} \times 20 \mathrm{~mm}$, Waters Co., Milford, MA, USA) for preconcentration with $99 \%: 1 \%$ / mobile phase A mobile phase B (mobile phase A: $\mathrm{H} 2 \mathrm{O}$ with $0.1 \% \mathrm{v} / \mathrm{v}$ formic acid, mobile phase B: acetonitrile with $0.1 \% \mathrm{v} / \mathrm{v}$ formic acid). Peptides were then separated with a BEH130 C18 column $(1.7 \mu \mathrm{m}, 100 \mu \mathrm{m}$ $\times 100 \mathrm{~mm}$, Waters Co., Milford, MA, USA) using an analytical gradient. The initial condition was $97.0 \% \mathrm{~A}$ and $3.0 \%$ B. Buffer B was ramped to $7.0 \%$ over 1 minute and then to $25 \%$ over 72.0 minutes. Over the next 10 minutes buffer B was increased from 25 to $45 \%$. The percent $B$ was then increased to $95 \%$ over 30 seconds and held for one minute. Buffer B was then returned to the initial condition of $3 \%$ and held for 10 minutes. The Thermo LTQ Orbitrap Velos ETD was operated in data dependent mode with the fifteen most intense ions determined from the MS spectra. Collision induced dissociation (CID) was used for peptide fragmentation. Dynamic exclusion was used with repeat counts, repeat duration, and exclusion duration of 1 , $30 \mathrm{~s}$, and 60s, respectively. Each run contained ap- 
proximately 22,000 scans. Samples were analyzed using an ion spray voltage and heated capillary temperature of $2.5 \mathrm{kV}$ and $300^{\circ} \mathrm{C}$, respectively. Data dependent acquisition was used in the mass spectrometry analysis, in which a high resolution $(60,000)$ MS survey scan (m/z 400 - 2000<tel:400\%20-\%202000>) with orbitrap (AGC: 1,000,000) was followed by an acquisition scheme consisting of CID mass spectrometry experiments. Mass spectrometry data were submitted to database searching using the MASCOT search engine. Database searches were performed against the Swiss Prot Rattus database, by using cysteine alkylation as static modification, methionine oxidation as dynamic modification and non-enzyme rule restrictions.

\section{Identification of F-Substance using rat cytokine antibody arrays}

The Rat Cytokine Array Panel A (Cat \# ARY008) from R\&D system (Minneapolis, MN) was used to probe cytokines in the substance isolated from stimulated and unstimualted rat muscles by following the procedures recommended by manufacturer. Briefly, the array membranes were first incubated in the block buffer for $1 \mathrm{hr}$. In the meantime, substances isolated from one stimulated or unstimulated muscle were mixed with the Detection Antibody Cocktail and incubated for 1 hour at room temperature. After removing the block buffer, the sample/antibody mixture were added to array membranes and incubated overnight at $4{ }^{\circ} \mathrm{C}$. After incubation, the membranes

A were washed 3 times with the Wash Buffer and then incubated the Steptavidin-HRP solution for 30 minutes at room temperature. The membranes were finally washed with Wash Buffer for 3 times and the bound antibodies were detected by chemiluminescence using the Immobilon ${ }^{\mathrm{TM}}$ Western Chemiluminescent HRP Substrate (Millipore, Billerica, MA). The membranes were quantified by densitometry. Statistical analysis was carried out by paired $t$ test.

\section{Results}

\section{Effect of F-Substance on muscle contraction upon electric stimulation}

To prepare the fatigue substance, rat femoris muscles were dissected and stimulated electrically as described in the Methods and Materials section. The lyophilized substance was reconstituted in cell culture media (RPMI with 10\% FBS) before use. To determine the "fatiguing" effect of the isolated substances, we injected the lyophilized sample into a fresh muscle and recorded the contraction pattern upon electrically stimulation. As shown in Figure 1A, the muscle injected with substances collected from stimulated muscles significantly diminished the contracting ability of the muscle (causing a fatigue effect) compared to the muscle injected with the same volume of saline solution (Figure 1B). This result indicates that the substances that we obtained from the stimulated muscle can cause fresh muscles to appear "fatigued".

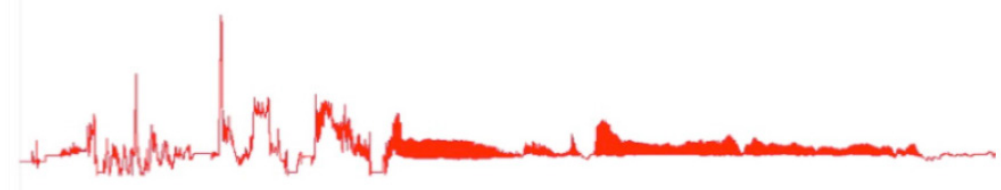

Muscle was injected with $1 \mathrm{ml}$ of $\mathrm{f}$-substance solution

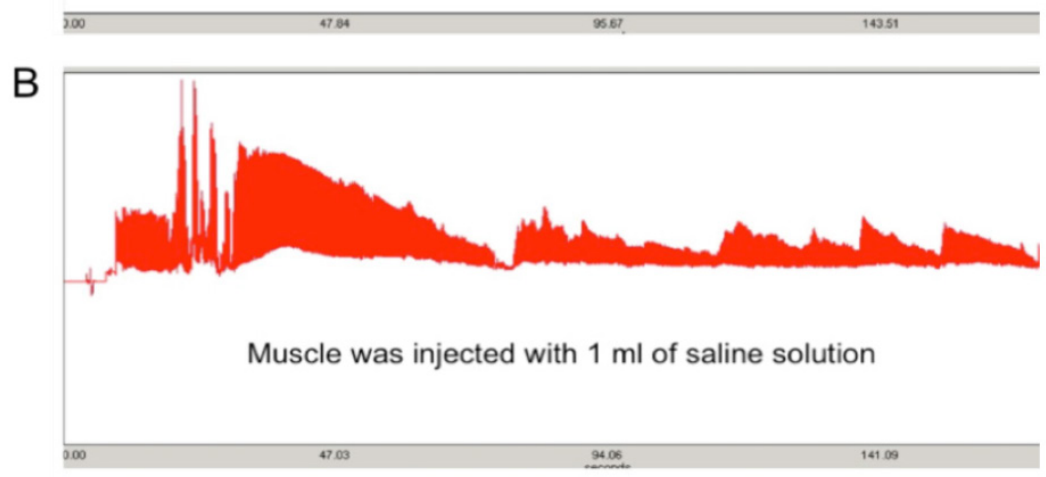

Figure I. The contraction ability of a muscle injected with I ml of substance extracted from stimulated muscle (A) is greatly diminished compared to that injected with the same volume of saline solution (B). Y axis: contraction force (grams); X-axis: time (seconds). 


\section{Effects of F-Substance on cancer cell growth}

To evaluate the anti-tumor activity of the substance released from the stimulated muscles, we measured its effect on the growth of two cancer cell lines, Mia PaCa-2 (a pancreatic cancer cell line) and MCF-7 (a breast cancer cell line). As shown in Figure 2A. While stimulated and unstimulated substances both showed inhibitory effect in the MCF-7 cells, the anti-proliferation activity of the substances obtained from the stimulated muscle was significantly higher than those obtained from the unstimulated muscles $(\mathrm{P}=0.02$ for $100 \mu \mathrm{L}$ volume using paired $t$ test). Furthermore, at low concentrations, only the substance from stimulated muscles showed significant time-dependent inhibition of MCF-7 cell growth (Figure 2B). Similar results were obtained for in Mia PaCa-2 cells (data not shown).

\section{Identification of proteins differentially pro- duced by the stimulated and unstimulated muscles using global proteomics analysis.}

Using the methodology described in the Material and Methods section, including a Waters nanoAcquity HPLC system running in ID mode combined with a Thermo LTQ Orbitrap Velos ETD operating in positive ion mode, peptides were separated and analyzed. The peptides that showed the significant difference between the stimulated and unstimulated muscles are listed in Supplementary Material: Table S1. A total of 106 proteins of which peptides showed differential spectral counts between stimulated and unstimulated samples in the analysis (a ratio of $\geq 2$ or $\leq 0.5$ between the stimulated sample and the unstimulated control, or only detected in either sample with a spectral counts $\geq 2$ ). Since the detection limit of the LC-MS system used in this study is in the single digit $\mathrm{ng} / \mathrm{mL}$ range, peptides with very abundance might have not been detected in the analysis. Further characterization of the peptides is needed to verify and validate their potential role in the anti-tumor activity of the F-substance.

\section{Cytokines that showed significant difference in abundance between the stimulated and un- stimulated muscle extracts.}

To further identify the active molecule(s) that are responsible for the antitumor activity of the fatigue substance, we used an antibody array containing antibodies against 29 different rat cytokines to detect cytokines in the substance (Figure 3). A total of 6 cytokines were detected and showed significant difference in levels in the samples isolated from unstimulated and stimulated muscles. Quantitation of the array membranes indicated that two of the 6 cytokines (IL-1 $\alpha$ and Thymus Chemokine) showed higher level in the samples isolated from unstimulated muscles and 4 showed higher level in the stimulated muscles, three (LIX, TIMP-1, and SICAM-1) of which showed greater than 2 fold difference and one (L-Selectin) showed 1.4 fold increased in the stimulated muscles (Figure 4). Paired $t$ test shows that the differences in the levels of cytokines between the stimulated and unstimulated muscles are highly significant $(\mathrm{P}$ values equal 0.006, 0.007, and 0.033 for LIX, TIMP-1, and sICAM-1, respectively).

To further confirm the array results, we performed Western blotting analysis for each of the 3 cytokines that showed the most significant difference in the arrays. As shown in Figure 5, the level of TIMP-1 is about 1.5 fold higher in the stimulated muscle extracts than in the unstimulated muscle.
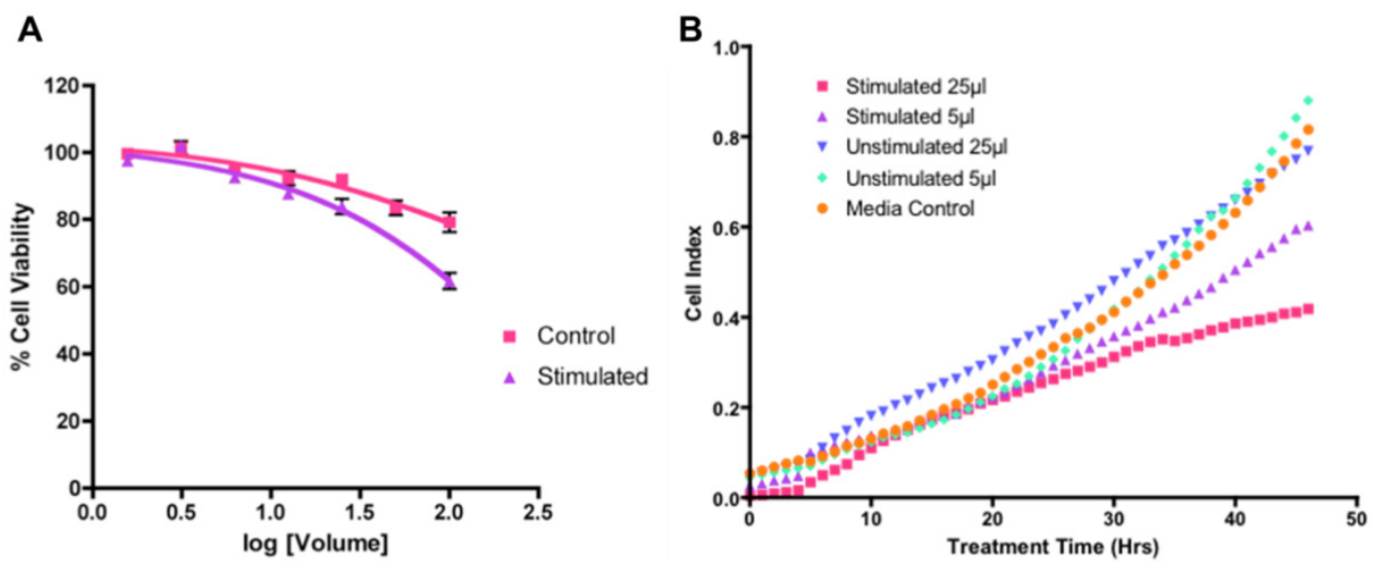

Figure 2. Growth inhibitory effect of the fatigue substance against MCF-7 cells. A. Concentration dependent effect of F-substance on MCF-7 cell growth determined by SRB assay. B. Real time cell growth effect of the F-Substance on MCF-7 using impedance measurements. 


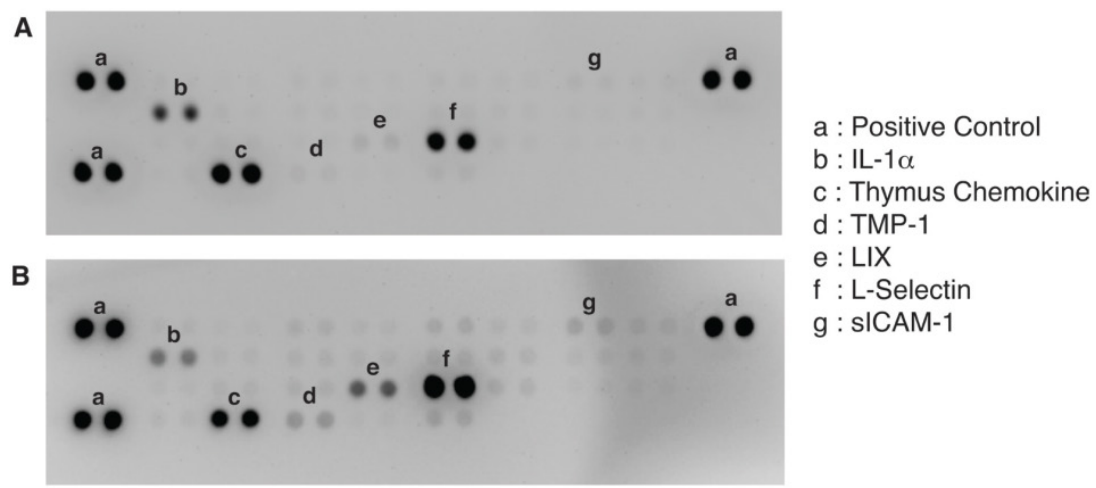

Figure 3. Identification of cytokines differentially produced by unstimulated (A) and stimulated (B) muscles. A Proteome Profiler Rat Cytokine Array Kit (R\&D Systems) that contains 29 different cytokines antibodies in duplicates was used to detect the cytokines contained in the extracts from muscles.

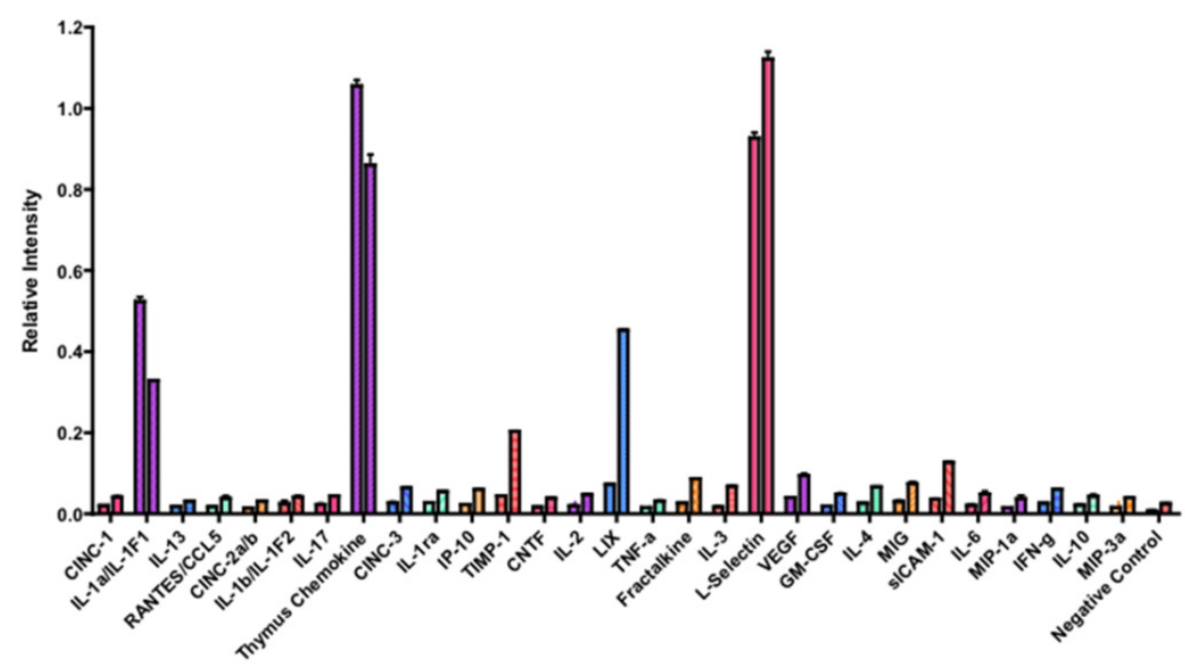

Figure 4. Quantification of the rat cytokines antibody array. The single intensity of each spot was quantified by densitometry. The ratio of the intensity between stimulated vs. unstimulated muscles was calculated. The cytokines that are the most significantly higher in the stimulated muscle are LIX, TIMP-I, and sICAM-I.

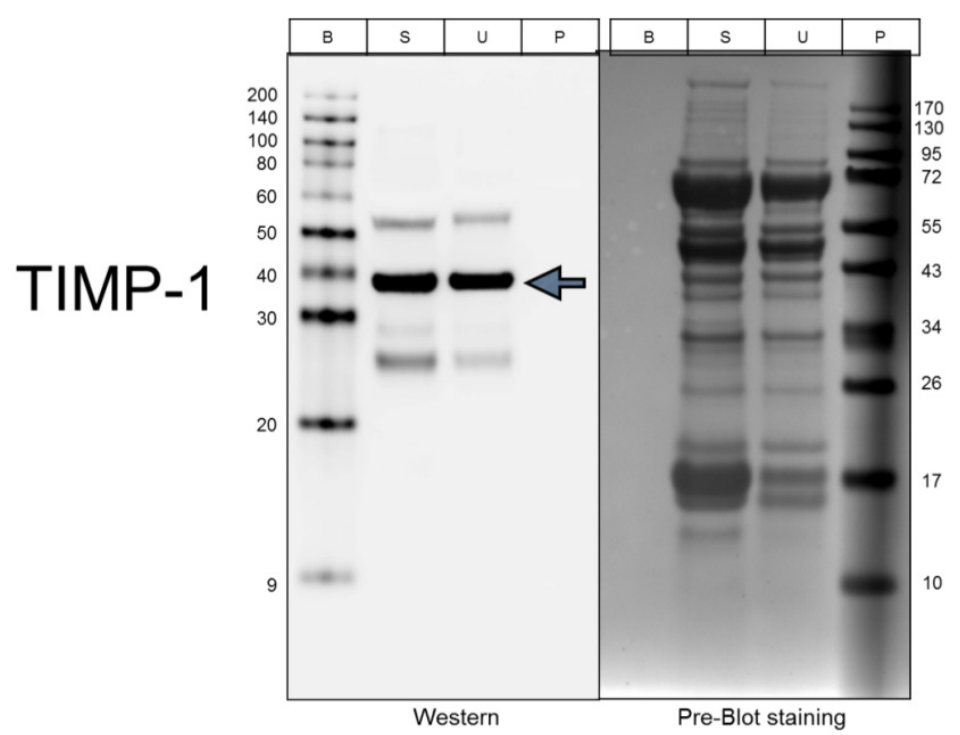

Figure 5. Validation of TIMP-I levels using Western Blotting. Lanes: B, biotinylated protein markers; S, Stimulated extract; U, Unstimulated extract; and $\mathrm{P}$, Prestained protein markers. 


\section{Discussion}

Between 1960 and 1978 our studies confirmed the anti-tumor effect of exercise and of F-Substance. However, with the technology available at the time, we were not able to fully identify its composition. Our original hypothesis about the anti-tumor effect of exercise has also been supported by several recent studies $(11,12,13)$. Thompson and colleagues stated that the effects of physical activity on the carcinogenic process "is consistent with an experiment reported over four decades ago indicating that contracting skeletal muscle produces a factor(s) that inhibits tumor cell growth (14). In this study we sought to identify the composition and activity of F-Substance. We determined that it contains cytokines, three of which were at significantly higher level in the substance isolated from the stimulated muscle than in the substance isolated from the unstimulated muscle.

Cytokines are known to play important roles in a number of biological processes, including innate immunity, apoptosis, angiogenesis, cell growth and differentiation. Many of these processes are involved in cancer pathogenesis and therapy (15). LIX (Lipopolysaccharide-induced CXC chemokine or CXCL5) is member of the CXC chemokine family which are potent neutrophil chemoattractants (16). TIMP-1 (Tissue inhibitor of metalloproteinases-1) is a naturally occurring inhibitor of metalloproteinases $(17,18,19)$. The TIMPs inhibit tumorigenesis, cellular invasion, metastasis and angiogenesis. However, they are also known to promote tumor growth and inhibit apoptosis. These paradoxical roles of TIMPs in tumor regression and progression have been attributed to the tissue microenvironment (20). sICAM-1 (Soluble intercellular adhesion molecule-1), a counter-receptor for the leukocyte integrin, lymphocyte function-associated antigen (LFA-1), has been reported to inhibit the interaction between $\mathrm{T}$ cells and tumors and, block NK cell-mediated toxicity, suggesting a cancer promoting role (21).

Further analysis of these cytokines will help determine whether or not these cytokines are responsible for the observed antitumor activity. Thompson et al. hypothesized that exercising muscles produce cytokines (14). Pedersen and co-workers suggested that "cytokines and other peptides that are produced, expressed, and released by muscle fibers and exert either paracrine or endocrine effects should be classified as myokines" (22). Pedersen further suggested that this "opens a whole new paradigm: skeletal muscle is an enodocrine organ which by contraction stimulates the production and release of cytokines" (23). Contracting skeletal muscle is also thought by some re- searchers to be the main source of IL- 6 within the body. Pedersen et al. reported that "exercise-induced plasma IL-6 concentration in almost exponential manner (22).

F-Substance demonstrates anti-tumor activity in both rat and human tumors (3). Our data indicates that the substance may exert its antitumor activity by signaling apoptosis in cancer cells. In 2007, Westerlind based a study on the original F-Substance research, confirming our hypothesis. The study also demonstrates apoptosis of cancer cells in response to treatment with fatigued muscle perfusates (24). Our current results are also consistent with Thompson's recent report showing apoptosis in breast cancer cells injected with extracts isolated from stimulated muscles (14). Westerlind and Thompson both speculate that muscle contraction causes the release of a factor into circulation which results in increased tumor cell apoptosis and smaller growing tumors. Additional research is needed to further characterize the antitumor activity of the substances including effect on human tumors and elucidate their mechanisms of action.

\section{Supplementary Material}

Table S1. http:/ / www.jcancer.org/v04p0343s1.pdf

\section{Acknowledgements}

We would like to thank Dr. David Azorsa for assistance with the ACEA system, Dr. Kendall Jensen for the SD9 stimulator, Dr. Cynthia Standley for the MP35 System, and Mario Sepulveda at TD2 for help with muscle dissection. We are also grateful to Ralph and Phillip Levitz for their financial support of our initial research and Joyce and Stanley Hoffman and Fern Mackour for their current support.

\section{Competing Interests}

The authors have declared that no competing interest exists.

\section{References}

1. Heilbrunn L. Dynamics of Living Protoplasm. New York, USA: Academic Press. 1956

2. Rashkis JA. Effect of Systemic Stress on Inhibition of Experimental tumors in Swiss Mice. Science, 1952;116:169-70

3. Hoffman SA, Paschkis KE, Cantarow AB. Exercise and tumor growth. Fed Proc 1960;19:396

4. Hoffman SA, Paschkis KE, Debias DA, et al. The influence of exercise on the growth of transplanted rat tumors. Cancer Res 1962;22:597-9

5. Hoffman SA, DeBias DA, Cantarow AB, et al. Effect of "Fatigue substance" on striated muscle contractions. Fed Proc 1968;27:443

6. Hoffman SA, DeBias DA, Cantarow AB, et al. The Effect of fatigue substance on transplanted rat tumors. Surg Forum 1968;19:85-87

7. Hoffman SA, Paxton DC, Wallace HW, Cantarow AB. F-Substance, Bio-assay method utilizing contracting muscles. Fed Proc 1969;28:824

8. Peck RL, Paxton DC, Cantarow AB, et al. Gel filtration of F-substance. Fed Proc. 1970;29:915 
9. Peck, RL, Cary, AL, and Debias, DA. Fatigue substance (F-substance): tumoristasis in relation to voltage regulation. Fed Proc 1973;32:803

10. Xie L, Kassner M, Munoz RM, et al. Kinome-wide siRNA screening identifies molecular targets mediating the sensitivity of pancreatic cancer cells to Aurora kinase inhibitors. Biochem Pharmacol 2012;83:452-61

11. Almeida P, Gomes-Filho A, Ferriera AJ, et al. Swim training suppresses tumor growth in mice. J Appl Physiol 2009;107: 261-265

12. Zhu Z, Jiang W, Sells JL, et al. Effect of nonmotorized wheel running on mammary carcinogenesis: circulating biomarkers, cellular processes, and molecular mechanisms in rats. Cancer Epidemiol Biomarkers Prev 2008;17:1920-1929

13. Zhu Z, Jiang W, McGinley JN et al. Energetics and mammary carcinogenesis: effects of moderate-intensity running and energy intake on cellular processes and molecular mechanisms in rats. J Appl Physiol 2008;106:911-918

14. Thompson HJ, Jiang W, Zhu Z. Candidate mechanisms accounting for effects of physical activity on breast carcinogenesis. IUBMB Life 2009;61:895-901

15. Dranoff G. Cytokines in cancer pathogenesis and cancer therapy. Nat Rev Cancer 2004;4:11-22

16. Chandrasekar B, Melby PC, Sarau HM, et al. Chemokine-cytokine cross-talk. The ELR+ CXC chemokine LIX (CXCL5) amplifies a proinflammatory cytokine response via a phosphatidylinositol 3-kinase-NF-kappa B pathway. J Biol Chem 2003;278:4675-86

17. Brew K, Dinakarpandian D, Nagase H. Tissue inhibitors of metalloproteinases: evolution, structure and function. Biochim Biophys Acta 2000;1477:267-83

18. Jiang Y, Goldberg ID, Shi YE. Complex roles of tissue inhibitors of metalloproteinases in cancer. Oncogene 2002;21:2245-52

19. Bourboulia D, Stetler-Stevenson WG. Matrix metalloproteinases (MMPs) and tissue inhibitors of metalloproteinases (TIMPs): Positive and negative regulators in tumor cell adhesion. Semin Cancer Biol 2010;20:161-8

20. Stetler-Stevenson WG. Tissue inhibitors of metalloproteinases in cell signaling: metalloproteinase-independent biological activities. Sci Signal 2008;1:re6

21. Witkowska AM, Borawska MH. Soluble intercellular adhesion molecule-1 (sICAM-1): an overview. Eur Cytokine Netw 2004;15:91-8

22. Pedersen BK, Akerstrom TC, Nielsen AR, et al: Role of myokines in exercise and metabolism. J Appl Physiol 2007;103:1093-8

23. Pedersen BK. Muscles and their myokines. J Exp Biol 2011;214:337-46

24. Westerlind KC. Muscle contraction arrests tumor growth. US Army Medical Research and Materiel Command Award Reports W81XWH-05-1-0464. 2007 